Case Report

\title{
Bone Marrow-Liver-Spleen Type of Large B-Cell Lymphoma Associated with Hemophagocytic Syndrome: A Rare Aggressive Extranodal Lymphoma
}

\author{
Kirill A. Lyapichev, ${ }^{1}$ Jennifer R. Chapman, ${ }^{1}$ Oleksii Iakymenko, ${ }^{1}$ Offiong F. Ikpatt, ${ }^{1}$ \\ Uygar Teomete, ${ }^{2}$ Sandra Patricia Sanchez, ${ }^{1}$ and Francisco Vega ${ }^{1,3}$ \\ ${ }^{1}$ Division of Hematopathology, Department of Pathology and Laboratory Medicine, \\ University of Miami and Sylvester Comprehensive Cancer Center, Miami, FL, USA \\ ${ }^{2}$ Department of Radiology, University of Miami and Sylvester Comprehensive Cancer Center, Miami, FL, USA \\ ${ }^{3}$ Division of Hematology-Oncology, Department of Medicine, University of Miami and Sylvester Comprehensive Cancer Center, \\ Miami, FL, USA
}

Correspondence should be addressed to Francisco Vega; fvega@med.miami.edu

Received 10 April 2017; Accepted 28 June 2017; Published 1 August 2017

Academic Editor: Kiyotaka Kawauchi

Copyright ( 2017 Kirill A. Lyapichev et al. This is an open access article distributed under the Creative Commons Attribution License, which permits unrestricted use, distribution, and reproduction in any medium, provided the original work is properly cited.

Recently, an unusual subtype of large B-cell lymphoma (LBCL) with distinctive clinicopathologic features has been recognized; it is characterized by involvement of bone marrow with or without liver and/or spleen, but no lymph node or other extranodal sites, usually associated with fever, anemia, and hemophagocytic lymphohistiocytosis (HLH). Because of this distinctive clinical presentation, it has been designated "bone marrow-liver-spleen" (BLS) type of LBCL. To date there is only one series of 11 cases of BLS type of LBCL with detailed clinical, pathologic, and cytogenetic data. Herein, we describe a case of BLS type LBCL presenting with associated HLH in a 73-year-old female. The bone marrow core biopsy showed cytologically atypical large lymphoma cells present in a scattered interstitial distribution and hemophagocytosis and infrequent large lymphoma cells were seen in the bone marrow aspirate smears. Circulating lymphoma cells were not seen in the peripheral blood smears. The patient underwent treatment with chemotherapy (R-CHOP) but unfortunately passed away 2 months after initial presentation. BLS type of LBCL is a very rare and clinically aggressive lymphoma whose identification may be delayed by clinicians and hematopathologists due to its unusual clinical presentation and pathologic features.

\section{Introduction}

Diffuse large B-cell lymphoma (DLBCL) is the most common non-Hodgkin lymphoma (NHL) accounting for almost $40 \%$ of cases [1]. DLBCL is an aggressive tumor that usually presents with rapidly enlarging lymph nodes or extranodal masses. Secondary bone marrow (BM) involvement occurs in approximately $10 \%-16 \%$ of patients with DLBCL, NOS; however $\mathrm{BM}$ is an unusual site to establish the initial diagnosis of DLBCL $[2,3]$. In addition to stage IV DLBCL, NOS, subtypes of DLBCL with relatively frequent BM involvement include intravascular large B-cell lymphoma (IVLBCL), splenic marginal zone lymphoma (splenic MZL), and Tcell/histiocyte-rich large B-cell lymphoma (THRLBCL) [1]. Recently a new subtype of DLBCL with frequent BM involvement and distinctive clinical and pathologic features has been recognized [4]. This lymphoma presents with BM involvement with or without involvement of the liver and/or spleen, often accompanied with severe anemia (100\%), thrombocytopenia, hemophagocytic lymphohistiocytosis (HLH) (64\%), and elevated lactate dehydrogenase (LDH) level in the absence of lymphadenopathy or other extranodal masses and with no prior history of lymphoma [4]. HLH is an uncommon a life-threatening disease of severe hyperinflammation 
caused by uncontrolled proliferation of activated lymphocytes and macrophages, characterized by proliferation of morphologically benign lymphocytes and macrophages that secrete high amounts of inflammatory cytokines. The current (2008) diagnostic criteria for HLH are a molecular diagnosis consistent with HLH (pathologic mutations of PRF1, UNC13D, or STX11) or fulfillment of five out of the eight criteria: (1) fever $\left(>100.4^{\circ} \mathrm{F},>38^{\circ} \mathrm{C}\right)$, (2) splenomegaly, (3) cytopenias (affecting at least two of three lineages in the peripheral blood: hemoglobin $<9 \mathrm{~g} / \mathrm{dL}$, platelets $<100 \times 10^{9}$ $/ \mathrm{L}$, or Neutrophils $\left.<1 \times 10^{9} / \mathrm{L}\right)$, (4) hypertriglyceridemia and/or hypofibrinogenemia ( $\leq 150 \mathrm{mg} / 100 \mathrm{ml}$ ), (5) ferritin $\geq$ $500 \mathrm{ng} / \mathrm{ml}$, (6) hemophagocytosis in the bone marrow, spleen, or lymph nodes, (7) low or absent natural killer cell activity, and (8) soluble CD25 (soluble IL-2 receptor) $>2400 \mathrm{U} / \mathrm{ml}$ (or per local reference laboratory) [5].

Because of its distinctive clinical presentation, this aggressive B-cell neoplasm has been named "bone marrow-liverspleen” (BLS) type of LBCL (LBCL-BLS). Here, we present a case of LBCL-BLS associated with haemophagocytic syndrome in a 73-year-old female and compare it with previously published cases. Due to the limited availability of published accounts of this rare entity, we believe it is important to document our case in order to add to our understanding of this rare but clinically aggressive lymphoma.

\section{Case Report}

2.1. Clinical Presentation. A 73-year-old Hispanic female was admitted to our institution with weakness, fever, thrombocytopenia $\left(70 \times 10^{9} / \mathrm{L}\right)$, neutropenia, elevated level of LDH (1010 $\mathrm{U} / \mathrm{L}$ ) and ferritin (>2000 $\mathrm{ng} / \mathrm{ml}$ ), and anemia ( $\mathrm{Hb} 6.3 \mathrm{~g} / \mathrm{dL})$ of unclear etiology that required blood transfusions. She had no significant past medical history except for diabetes mellitus (DM).

Computed tomography (CT) scan at the time of admission revealed no evidence of mediastinal, hilar, or other systemic lymphadenopathy but showed marked enlargement of liver and spleen (Figure 1). The spleen showed numerous heterogeneous areas of hypoenhancement, ranging between 2 and $5 \mathrm{cms}$, consistent with a neoplasm and suspicious for lymphoma. The adrenal glands were grossly within normal limits. Lung windows demonstrated patency of the trachea and main bronchi. No definite discrete pulmonary nodules or masses were identified. Osseous windows showed no definite lytic, sclerotic, or destructive lesions.

2.2. Pathologic Findings. A BM biopsy was performed to investigate the etiology of the symptomatic anemia and thrombocytopenia. The BM core biopsy demonstrated a marrow cellularity of $60 \%$ with scattered large atypical lymphoid cells (Figure 2(a)). These cells were either scattered individually or formed loose clusters and represented approximately $10 \%$ of the total marrow cellularity (Figure 2(b)). Large clusters or sheets of lymphoma cells were not seen. Immunohistochemical studies showed that the tumor cells expressed pan-B-cell markers CD20 and CD79a and were negative for CD3, CD4, CD8, CD56, CD57, PD1, CD30, TdT, glycophorin, MPO, and CD117 (Figures 2(b) and 2(c)).

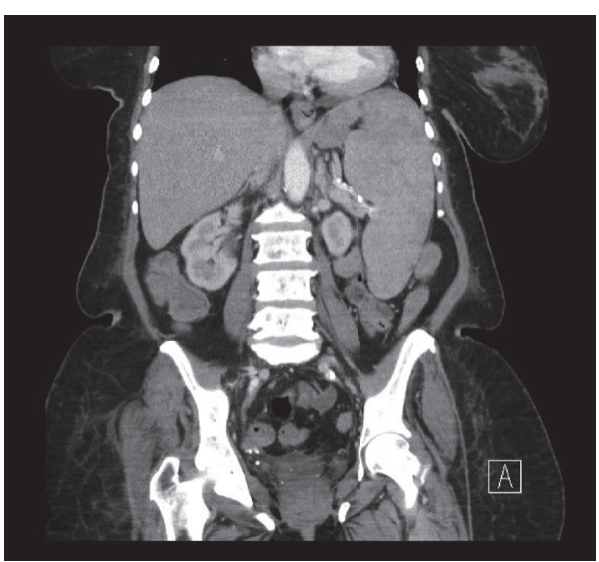

Figure 1: Abdominal and pelvic CT scan. Marked hepatomegaly and splenomegaly are identified. Spleen demonstrates numerous areas of heterogeneous hypoenhancement.

CD20 and CD79a highlighted the interstitial distribution of the tumor cells. Tumor cells inside the marrow sinusoids were not seen (Figure 2(b)). Similarly, a CD34 immunostain and dual PAX5 with CD34 immunostains failed to show tumor cells inside the vessels. The lack of intrasinusoidal and intravascular tumor excluded the diagnosis of IVLBCL (Figures 2(d) and 3(a) and Table 2). As per the Hans algorithm based on the expression of CD10, BCL6, and MUM1, our case was classified into the nongerminal center B-cell-like (nonGCB) subgroup. IHC showed CD10 reactivity in less than $30 \%$ of tumor cells and MUM1 reactivity in more than $30 \%$ of tumor cells (Figures 3(b) and 3(c)). Additionally, BM biopsy was stained for MYC and for BCL2 and showed a subset of positive tumor cells (Figures 4(a) and 4(b)). The lymphoma cells were negative for Epstein-Barr virus-encoded RNAs (EBER). Small reactive T-cells were distributed throughout the marrow and consisted of a mixed population of CD4 and CD8 positive T-cells. Importantly, nodules rich in Tcells associated with the lymphoma cells were not seen and therefore the diagnosis of THRLBCL was also excluded (Table 2).

Individual large, atypical lymphoid cells were also recognized on the aspirate smears (Figure 2(e)). In addition, there were numerous histiocytes demonstrating hemophagocytosis throughout the aspirate smears (Figure 2(f)). Mild trilineage dyspoiesis was seen. Some of the dyspoietic granulocytes had toxic changes. A peripheral blood smear showed no circulating lymphoma cells.

Flow cytometry immunophenotypic analysis was performed on BM specimen and was negative for the presence of monoclonal B cells or increased blasts. Conventional karyotyping was negative for any clonal abnormalities and showed a diploid female karyotype, 46;XX [6]. Immunoglobulin heavy chain (IgH) and kappa light chain PCR assays performed in BM aspirate material confirmed the presence of a monoclonal B-cell population. This discrepancy between flow cytometry results and molecular analysis could be explained by poor representation of the tumor cells during flow cytometry analysis and high sensitivity of PCR assay. 


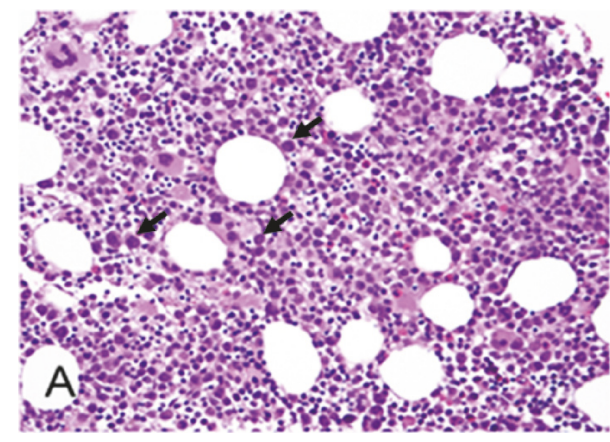

(a)

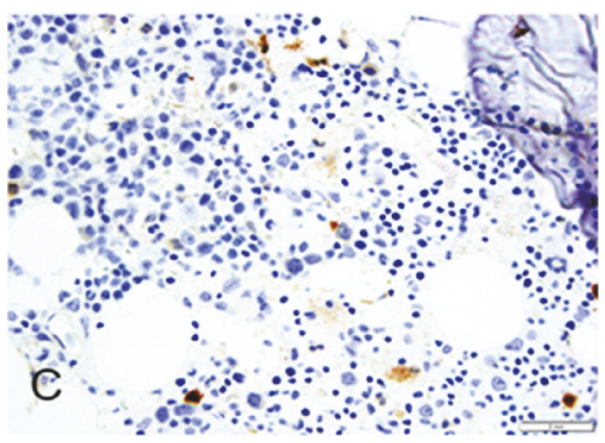

(c)

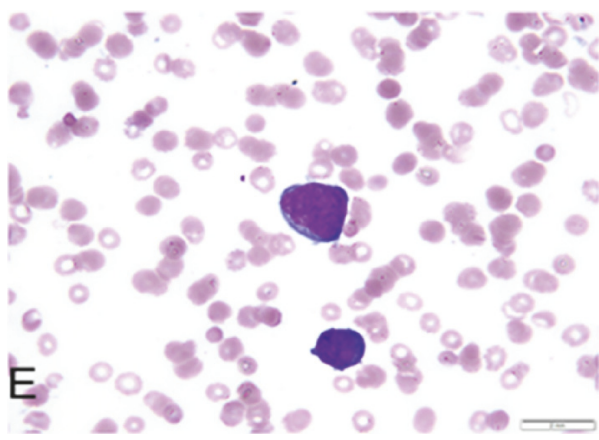

(e)

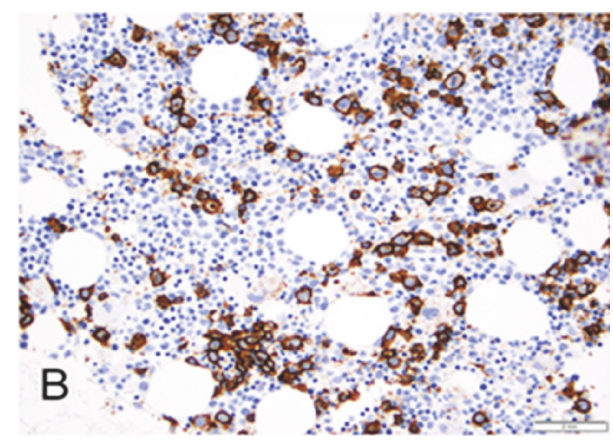

(b)

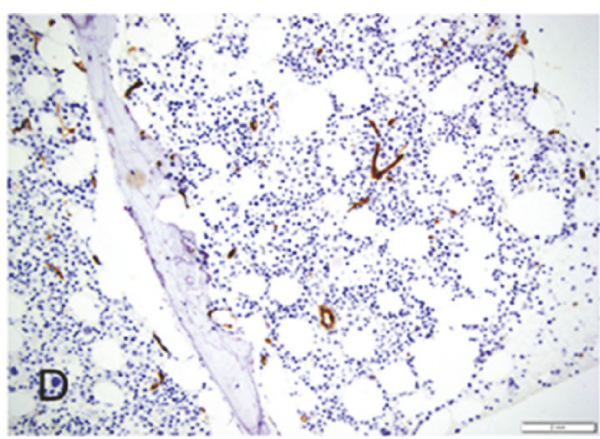

(d)

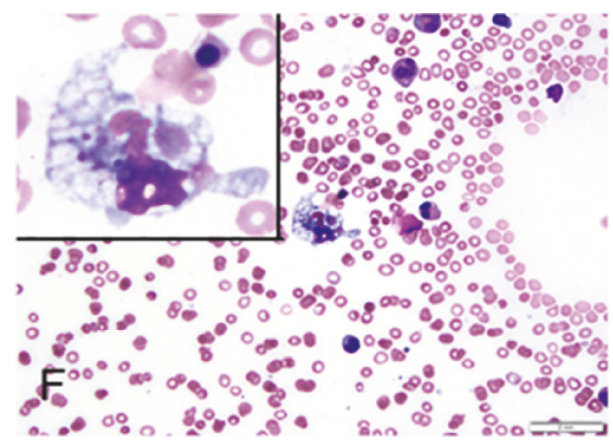

(f)

FIGURE 2: Histopathologic features. BM biopsy shows patchy interstitial infiltration of large lymphoma cells (arrows) without sinusoidal involvement (H\&E; 400x) (a). By immunohistochemistry (IHC), the tumor cells are positive for CD20 (400x) (b) and negative for CD30 (400x) (c). CD34 highlights the capillary endothelial cells and confirms the lack of intravascular involvement by tumor cells (400x) (d). Lymphoma cells are large in size with large nuclei, high nuclear to cytoplasmic ratios, and open chromatin with one to several prominent nucleoli (Wright Geimsa; 100x) (e). BM aspirate smear shows histiocytic hemophagocytosis characterized by RBCs engulfing by histiocytes (Wright Geimsa; 100x) (f).

T-cell receptor (TCR) gamma PCR assay was negative for Tcell clonality.

Combining the pathologic findings with the clinical presentation, the patient was diagnosed with LBCL-BLS type. The neoplasm was associated with severe anemia, hepatosplenomegaly, and HLH syndrome. A biopsy of the spleen and the liver was not performed but based on the radiological studies both organs were considered involved by lymphoma.

2.3. Treatment and Clinical Course. After diagnosis, treatment with R-CHOP was immediately started. Approximately two months (51 days) after initiation of therapy the patient developed septic shock due to chemotherapy induced neutropenia and passed away.

\section{Discussion}

LBCL-BLS type is distinctive aggressive LBCL with characteristic clinicopathological features and aggressive clinical behavior that has been relatively recently recognized [4]. Patients usually present with fever, anemia of unclear etiology, thrombocytopenia, and increased LDH. On imaging studies splenomegaly or hepatosplenomegaly is typical and many small (usually less than $3 \mathrm{~cm}$ ) hypodense nodules can be identified. Bone marrow biopsy usually 


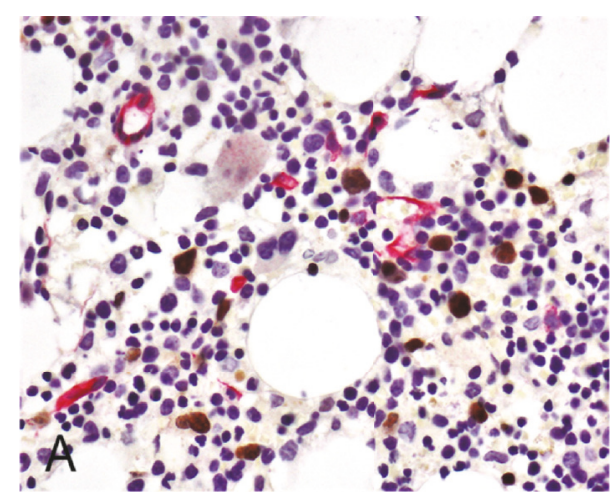

(a)

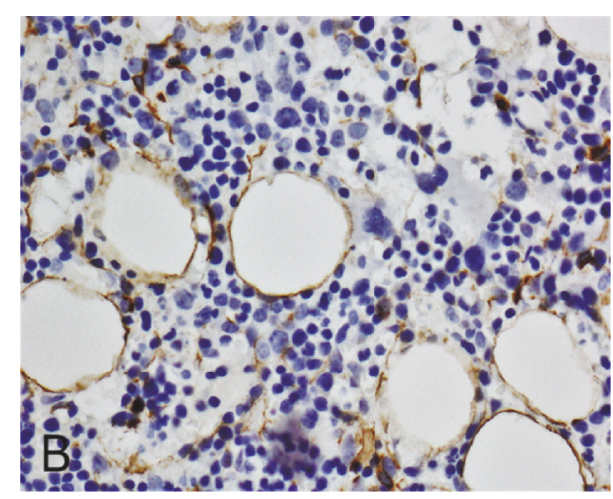

(b)

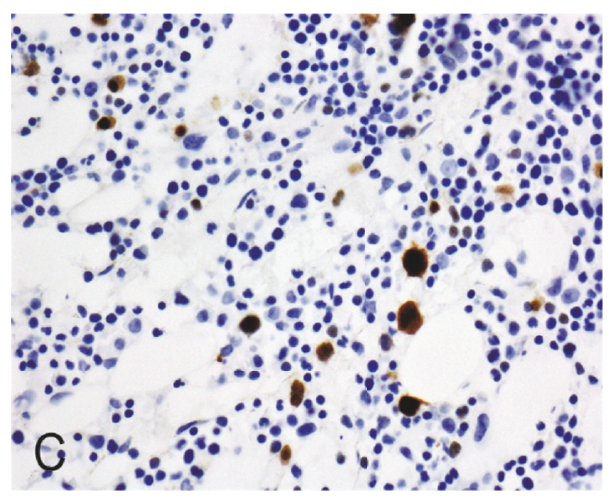

(c)

FIGURE 3: Histopathologic features. BM biopsy shows patchy interstitial infiltration of large, PAX5 positive lymphoma cells without sinusoidal (CD34 positive) involvement (400x) (a). By IHC, less than 30\% of tumor cells are positive for CD10 (400x) (b) and more than $30 \%$ positive for MUM1 (400x) (c).

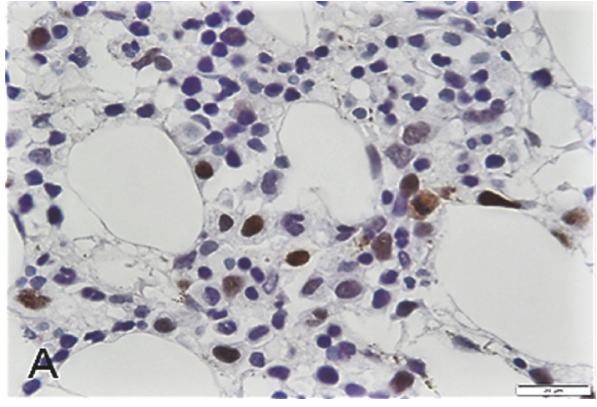

(a)

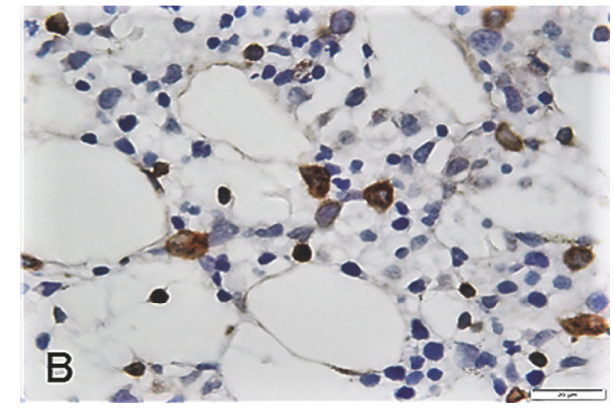

(b)

Figure 4: Histopathologic features. BM biopsy shows a subset of the tumor cells positive for MYC (100x) (a) and for BCL2 (100x) (b).

reveals hemophagocytosis and an interstitial distribution of large lymphoma cells with a mature B-cell phenotype. Importantly, the lymphoma cells are not involving vascular lumina or sinusoids, differentiating this presentation from that of intravascular large B-cell lymphoma. To date, there are no specific genetic abnormalities and viral associations identified.

Nonnodal, primary LBCL involving BM with or without involvement of liver and/or spleen and no other extranodal involvement has been variably published in the literature [716]. However, given that this type of LBCL is not currently defined in the WHO classification, previous studies which included different types of lymphomas have been inconsistent in their reports of pathologic features and clinical outcomes. These previous reports seem to describe clinically similar lymphomas presenting with marrow involvement with separation into different categories based on additional site of involvement (BM only, spleen and BM or spleen, liver and $\mathrm{BM})$.

To find previously reported cases of LBCL-BLS similar to ours, during the literature search we selected only studies of LBCL with initial manifestation in the BM, liver, and/or 
spleen (BLS type) that lacked any lymph node or other extranodal involvement and included genetic and survival information. Additionally, to make our search more specific we excluded diagnoses of IVLBCL, splenic marginal cell lymphoma with transformation, primary bone LBCL, and THRLBCL and used "Large B-cell lymphoma, bone marrow, liver, and spleen" as the keywords during literature search by PubMed. Using these criteria, we found only two publications that seemed similar to the case of LBCL-BLS that we describe $[4,14]$.

One of these, a study by Iioka et al. [14], describes 10 cases of DLBCL confined to BM, spleen, and liver, as evidenced by the uniformly increased uptake of fluorodeoxyglucose (FDG) on positron emission tomography combined with computed tomography (PET/CT). They retrospectively reviewed the clinical records of patients with aggressive B-cell lymphoma who were diagnosed and treated in their institution between 2011 and 2015. The inclusion criteria were as follows: patients whose clinical stage was determined on the basis of a FDGPET/CT imaging study; patients whose disease showed the uniformly increased uptake of the tracer in the BM with or without diffuse uptake in the spleen and/or liver, but lacked uptake in the lymph nodes; and patients in whom a BM examination was performed for the diagnosis. Burkitt lymphoma, mantle cell lymphoma, the transformation of low-grade B-cell lymphomas, and any leukemia/lymphoma entity known to arise primarily in the BM were excluded. This study might represent 10 cases of LBCL-BLS but the authors do not specifically discriminate it from IVLBCL [14].

The BM biopsies were available in 9 out of 10 cases. Histology revealed lymphoma cells that were large with a moderate amount of cytoplasm, large vesicular nuclei, and one or more prominent nucleoli. Immunohistochemistry showed that lymphoma cells were positive for CD20 and CD79a. CD5 was positive in two cases showing the intrasinusoidal infiltration pattern; CD5 positivity was confirmed by flow cytometry. BCL2 was positive in eight cases. Per the Hans algorithm based upon the expression of CD10, BCL6, and MUM1, all cases were classified into the nongerminal center B-cell-like (non-GCB) subgroup. But they did not discriminate their cases from IVLBCL (did not perform CD34 IHC, to see intrasinusoidal involvement). Additionally, authors agreed that they will need to perform comparative studies incorporating histopathology and findings on FDG-PET/CT due to distinguish LBCL-BLS from IVLBCL. A different study by Yeh et al. reported detailed clinical, pathologic, and cytogenetic data in a series of LBCL-BLS whose clinical and histopathologic features were similar to those of our case [4]. In looking at the histopathologic features and clinical outcomes of the patients in the Yeh et al. series and our own, LBCL-BLS type seems to be a distinct clinicopathologic type of LBCL which has an aggressive clinical course and poor survival (Table 1). These lymphomas are characterized by variable age at presentation: $26-80$ years, predominantly male population (M:F, 8:4), fever (100\%), anemia (100\%), thrombocytopenia (83\%), increased LDH level (92\%), bone marrow involvement (100\%) with HLH syndrome (67\%), nongerminal center phenotype (83\%, as established by Hans algorithm), splenomegaly (100\%), and hepatomegaly (25\%) in the absence of lymphadenopathy and with no prior history of lymphoma. Additionally, it seems that the patient's survival rate depends at least in part on rapid lymphoma diagnosis and adequate and prompt treatment. Five patients (42\%) in the previously reported series died in the first two and a half weeks after diagnosis (from 3 to 17 days); the patient we present died of lymphoma-related sepsis after only 51 days despite rapid lymphoma diagnosis and treatment initiation.

In Table 2 we compare LBCL-BLS as defined in this manuscript (LBCL involving BM, liver, and/or spleen but lacking intravascular or sinusoidal involvement and lacking nodal or other extranodal disease) with intravascular large Bcell lymphomas (Western and Asian types) and THRLBCL [1]. The lymphoma most similar to LBCL-BLS that is currently WHO-defined is IVLBCL, at least from the clinical perspective. Splenic MZL and THRLBCL might have similar clinical presentation too but usually they are very different pathohistologically and missing HLH. The lymphoma cells in splenic MZL are smaller and usually infiltrate BM with mixed pattern: combining nodular, paratrabecular, or diffuse with a distinctive intrasinusoidal component. Chromosomal translocation involving CDK6 gene at 7q21 as well as allelic loss of $7 \mathrm{q} 31-32$ is seen in a significant subset of the splenic MZL cases; NOTCH 2 and KLF2 mutations also might be present [17]. THRLBCL commonly involves liver, spleen, and bone marrow in addition to lymph node. In bone marrow, THRLBLC is characterized by multinodular aggregates rich in T-cells often associated with histiocytes and scattered large atypical lymphoid cells.

IVLBCL is a rare type of extranodal LBCL characterized by the selective growth of lymphoma cells within the lumina of capillaries and small/medium sized vessels. There are two IVLBCL subtypes: Western type characterized by symptoms related to the main organ involvement, predominantly central nervous system (CNS) or skin, and an Asian type in which the patients present with multiorgan failure, hepatosplenomegaly, pancytopenia, fever, B symptoms, and HLH. The second type (Asian type) of IVLBCL is closer in clinical presentation (pancytopenia, HLH, and skin and central nervous system involvements are uncommon) to LBCL-BLS with the pathologic difference being that lymphoma cells have a striking intravascular and intrasinusoidal distribution in Asian type IVLBCL. B symptoms are very common (55-76\%) and lymphoma cells are more often of activated B-cell type in both types of IVLBCL as well as in LBCL-BLS [1]. Polymerase chain reaction (PCR) studies in both entities show negative results for Epstein-Barr virus (EBV) and human herpesviruses 6 and 8 (HHV6 and HHV8) infections in vast majority of cases. It is unclear at this time whether IVLBCL, particularly Asian type, and LBCLBLS type, as reported herein, are variants of the same highgrade form of LBCL. A high incidence of HLH is also an important and characteristic feature of both IVLBCL and LBCL-BLS type. Although the precise mechanism underlying this lymphoma-associated HLH syndrome remains unclear, overproduction of TNF- $\alpha$, INF- $\gamma$, IL-1a, IL-6, IL-10, and other cytokines by nonfunctional cytotoxic T/NK-cells as well as macrophage hyperactivation is thought to play a crucial role [18-20]. 


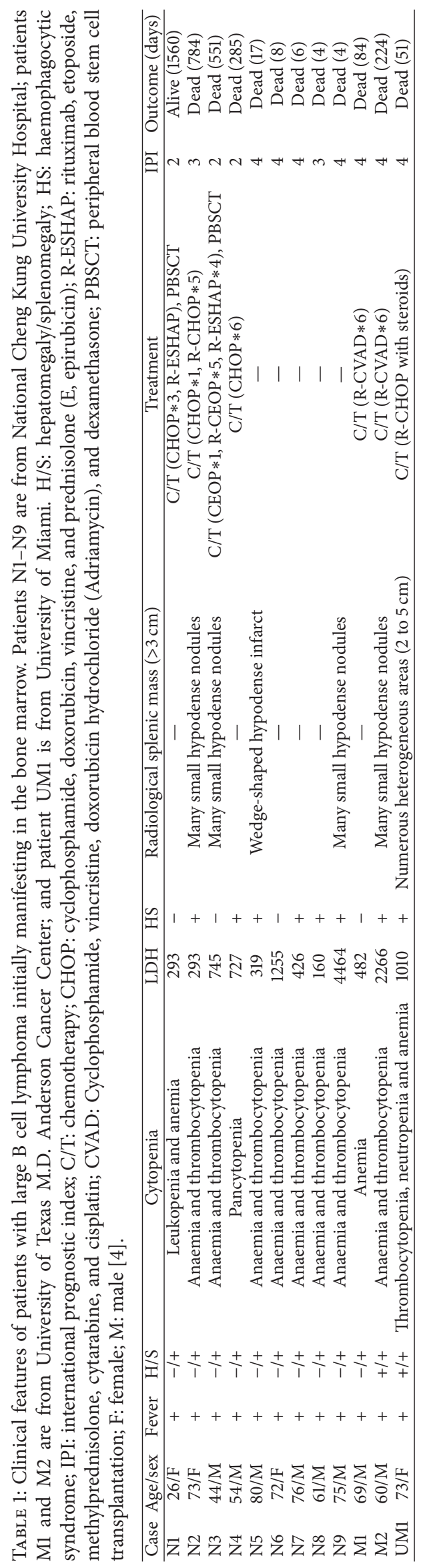


TABLE 2: Differential diagnosis of LBCL-BLS and subtypes of IVLBCL and TCHRLBCL.

\begin{tabular}{|c|c|c|c|c|}
\hline & LBCL-BLS & IVLBCL (Western type) & IVLBCL (Asian type) & TCHRLBCL \\
\hline $\mathrm{CD} 20$ & + & + & + & + \\
\hline CD79a & + & + & + & + \\
\hline CD3 & - & - & - & Background T cells \\
\hline CD5 & - & $38 \%+$ & $38 \%+$ & Background T cells \\
\hline EBV & - & - & - & + \\
\hline HLH & + & $+/-$ & + & - \\
\hline Peripheral lymph node involvement & - & - & - & + \\
\hline Involvement of vascular lumina by lymphoma cells & - & + & + & - \\
\hline Specific Alterations & None & None & $19 \mathrm{q} 13$ and $8 \mathrm{p} 21$ & - \\
\hline Organ of involvement & $\begin{array}{l}\text { BM, liver } \\
\text { and/or } \\
\text { spleen }\end{array}$ & CNS or skin & $\mathrm{BM}$ & Liver, spleen, BM \\
\hline T cell rich lymphoid nodules & - & - & - & + \\
\hline $\mathrm{HCV}$ infection association & - & - & - & - \\
\hline Bone marrow, rich in $\mathrm{T}$ cell nodules & - & - & - & + \\
\hline
\end{tabular}

In addition to the difference in lymphoma cell distribution (intravascular versus BM interstitial), the Asian type of IVLBCL and LBCL-BLS type have also not yet shown overlapping molecular genetic features, supporting that these two lymphoma types are different. Specifically, Asian type IVLBCL has been associated with abnormalities of $19 \mathrm{q} 13$ and $8 \mathrm{p} 21$, which have not been found in LBCL-BLS type thus far $[4,6,21]$. LBCL-BLS type, alternatively, has shown multiple chromosomal defects including $\mathrm{t}(8 ; 14)(\mathrm{q} 24 ; \mathrm{q} 32), \operatorname{dup}(14)(\mathrm{q} 24 ; \mathrm{q} 32), \operatorname{inv}(14)(\mathrm{q} 32)$, $\mathrm{t}(3 ; 6)$, trisomy 18, add(19)(p13), del(8)(p22), del(3)(q21), and $\operatorname{add}(7)(\mathrm{p} 22)[4,22,23]$. However, given the limited number of available cases in the literature at this time, additional studies are needed with specific comparisons of these two lymphoma types to determine their relatedness, or lack thereof. Finally, lack of CD29 ( $\beta-1$ integrin) and CD54 (ICAM-1) in IVLBCL may be potentially useful diagnostic feature. Defects (downregulation) in these protein receptors on the lymphoma cells might prevent the neoplasm from invasion through vascular wall and contribute to the intravascular location.

\section{Conclusion}

We describe an unusual and clinically aggressive extranodal large B-cell lymphoma involving the BM, spleen, and liver (LBCL-BSL). This lymphoma appears to be a distinct but rare and controversial entity that may be a specific type of extranodal DLBCL, NOS [1]. However, aside from lymphoma cell distribution within vascular spaces and marrow sinusoids, this lymphoma has clinical features similar to those of Asian type IVLBCL. Importantly, the clinical presentation and disease course are particularly severe with rapid disease progression and high mortality rate during first weeks to months after initial symptoms. Overlapping clinical and morphological features can make it challenging to differentiate LBCL-BLS from more common lymphomas including splenic marginal zone lymphoma with large cell or prolymphocytic transformation, THRLBCL and, particularly, IVLBCL (Asian type). Gene expression profiling studies comparing these entities may help in developing an understanding of the biology of these lymphomas and their relatedness as well as in predicting effective treatment protocols.

\section{Conflicts of Interest}

The authors declare that there are no conflicts of interest regarding the publication of this paper.

\section{References}

[1] N. L. J. Harris, S. A. Pileri, H. Stein, J. Thiele, and J. W. Vardiman, "WHO classification of tumours of haematopoietic and lymphoid tissues," Vol. 2. 2008.

[2] L. H. Sehn, D. W. Scott, M. Chhanabhai et al., "Impact of concordant and discordant bone marrow involvement on outcome in diffuse large B-cell lymphoma treated with R-CHOP," Journal of Clinical Oncology, vol. 29, no. 11, pp. 1452-1457, 2011.

[3] R. Chung, R. Lai, P. Wei et al., "Concordant but not discordant bone marrow involvement in diffuse large B-cell lymphoma predicts a poor clinical outcome independent of the International Prognostic Index," Blood, vol. 110, no. 4, pp. 1278-1282, 2007.

[4] Y.-M. Yeh, K.-C. Chang, Y.-P. Chen et al., "Large B cell lymphoma presenting initially in bone marrow, liver and spleen: An aggressive entity associated frequently with haemophagocytic syndrome," Histopathology, vol. 57, no. 6, pp. 785-795, 2010.

[5] J.-I. Henter, A. Horne, M. Aricó et al., "HLH-2004: diagnostic and therapeutic guidelines for hemophagocytic lymphohistiocytosis," Pediatric Blood \& Cancer, vol. 48, no. 2, pp. 124-131, 2007.

[6] T. Murase, S. Nakamura, K. Kawauchi et al., "An Asian variant of intravascular large B-cell lymphoma: clinical, pathological and cytogenetic approaches to diffuse large B-cell lymphoma associated with haemophagocytic syndrome," British Journal of Haematology, vol. 111, no. 3, pp. 826-834, 2000. 
[7] C. G. Roth and K. K. Reichard, "Subtle bone marrow involvement by large B-cell lymphoma with pronormoblast-like morphology and prominent but not exclusive sinusoidal distribution," American Journal of Blood Research, vol. 2, no. 2, pp. 113118, 2012.

[8] D. Kajiura, Y. Yamashita, and N. Mori, "Diffuse large B-cell lymphoma initially manifesting in the bone marrow," American Journal of Clinical Pathology, vol. 127, no. 5, pp. 762-769, 2007.

[9] C. L. Alvares, E. Matutes, M. A. Scully et al., "Isolated bone marrow involvement in diffuse large B cell lymphoma: A report of three cases with review of morphological, immunophenotypic and cytogenetic findings," Leukemia and Lymphoma, vol. 45, no. 4, pp. 769-775, 2004.

[10] P. Bhagat, M. U. S. Sachdeva, P. Sharma et al., "Primary bone marrow lymphoma is a rare neoplasm with poor outcome: Case series from single tertiary care centre and review of literature," Hematological Oncology, vol. 34, no. 1, pp. 42-48, 2016.

[11] Y. Yamashita, D. Kajiura, L. Tang et al., "XCR1 expression and biased VH gene usage are distinct features of diffuse large B-cell lymphoma initially manifesting in the bone marrow," American Journal of Clinical Pathology, vol. 135, no. 4, pp. 556-564, 2011.

[12] W. G. Morice, F. J. Rodriguez, J. D. Hoyer, and P. J. Kurtin, "Diffuse large B-cell lymphoma with distinctive patterns of splenic and bone marrow involvement: Clinicopathologic features of two cases," Modern Pathology, vol. 18, no. 4, pp. 495-502, 2005.

[13] A. Martinez, M. Ponzoni, C. Agostinelli et al., "Primary bone marrow lymphoma: An uncommon extranodal presentation of aggressive non-hodgkin lymphomas," American Journal of Surgical Pathology, vol. 36, no. 2, pp. 296-304, 2012.

[14] F. Iioka, G. Honjo, T. Misaki et al., "A unique subtype of diffuse large B-cell lymphoma primarily involving the bone marrow, spleen, and liver, defined by fluorodeoxyglucose-positron emission tomography combined with computed tomography," Leukemia and Lymphoma, vol. 57, no. 11, pp. 2593-2602, 2016.

[15] H. Chang, Y.-S. Hung, T.-L. Lin et al., "Primary bone marrow diffuse large B cell lymphoma: A case series and review," Annals of Hematology, vol. 90, no. 7, pp. 791-796, 2011.

[16] J. A. Strauchen, "Primary bone marrow B-cell Lymphoma: report of four cases," Mount Sinai Journal of Medicine, vol. 70, no. 2, pp. 133-138, 2003.

[17] N. Martínez, C. Almaraz, J. P. Vaqué et al., "Whole-exome sequencing in splenic marginal zone lymphoma reveals mutations in genes involved in marginal zone differentiation," Leukemia, vol. 28, no. 6, pp. 1334-1340, 2014.

[18] J.-D. Lay, C.-J. Tsao, J.-Y. Chen, M. E. Kadin, and I.-J. Su, "Upregulation of tumor necrosis factor- $\alpha$ gene by EpsteinBarr virus and activation of macrophages in Epstein-Barr virus-infected $\mathrm{T}$ cells in the pathogenesis of hemophagocytic syndrome," Journal of Clinical Investigation, vol. 100, no. 8, pp. 1969-1979, 1997.

[19] B. J. Chen, B. Chapuy, J. Ouyang et al., "PD-L1 expression is characteristic of a subset of aggressive B-cell lymphomas and virus-associated malignancies," Clinical Cancer Research, vol. 19, no. 13, pp. 3462-3473, 2013.

[20] K. Devitt, J. Cerny, B. Switzer et al., "Hemophagocytic lymphohistiocytosis secondary to T-cell/histiocyte-rich large B-cell lymphoma," Leukemia Research Reports, vol. 3, no. 2, pp. 42-45, 2015.

[21] C. Shimazaki, T. Inaba, K. Shimura et al., "B-cell lymphoma associated with haemophagocytic syndrome: A clinical, immunological and cytogenetic study," British Journal of Haematology, vol. 104, no. 4, pp. 672-679, 1999.
[22] H. Geyer, N. Karlin, B. Palen, and R. Mesa, "Asian-variant intravascular lymphoma in the African race," Rare Tumors, vol. 4, no. 1, pp. 26-29, 2012.

[23] H. Khoury, V. S. Lestou, R. D. Gascoyne et al., "Multicolor karyotyping and clinicopathological analysis of three intravascular lymphoma cases," Modern Pathology, vol. 16, no. 7, pp. 716-724, 2003. 


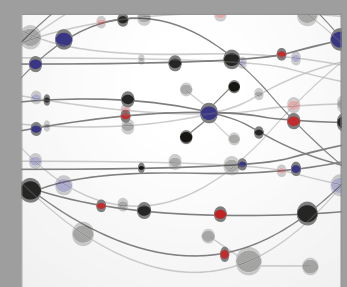

The Scientific World Journal
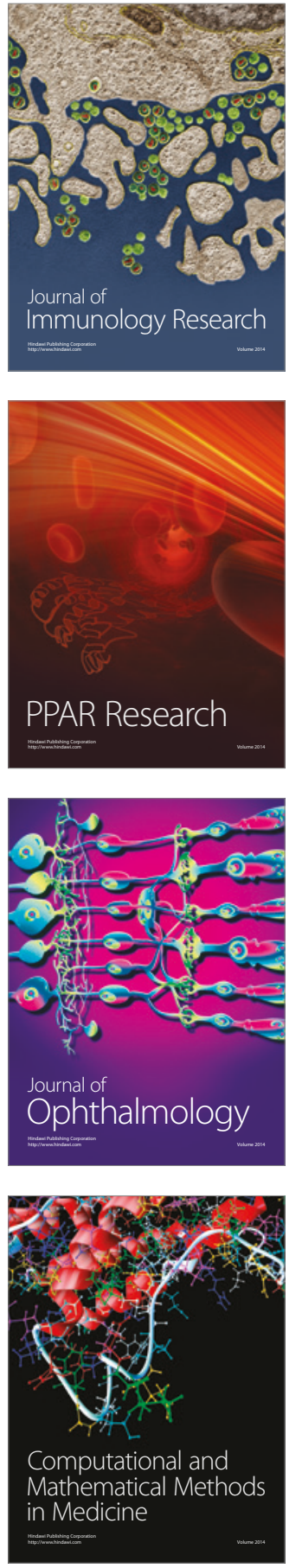

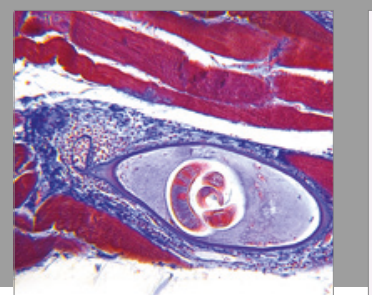

Gastroenterology Research and Practice
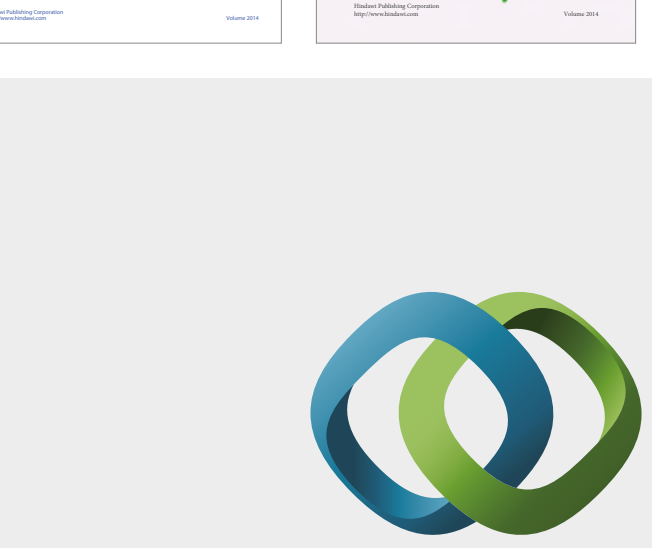

\section{Hindawi}

Submit your manuscripts at

https://www.hindawi.com
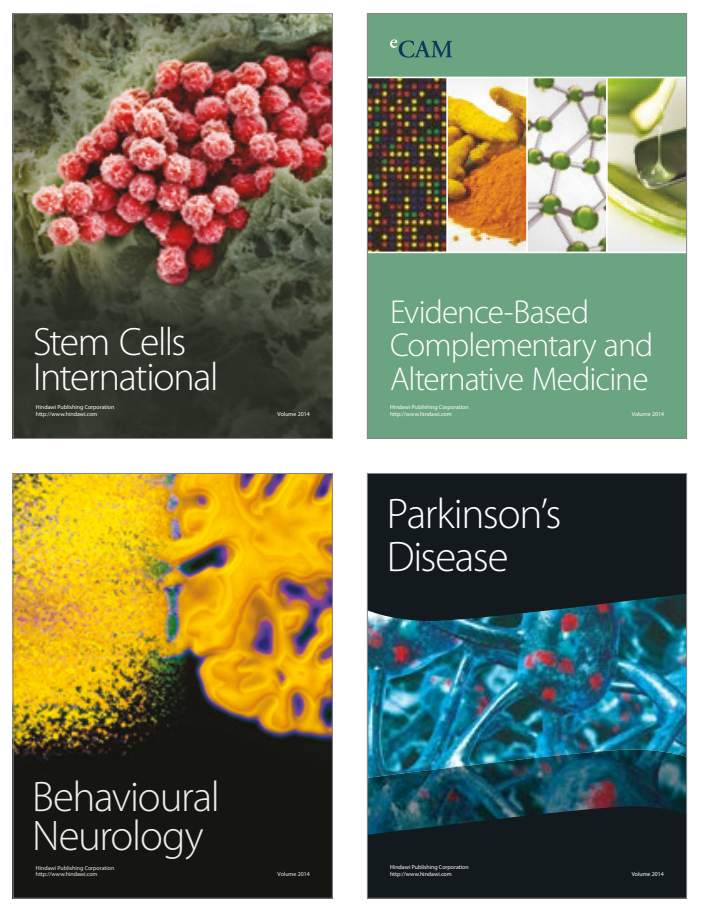
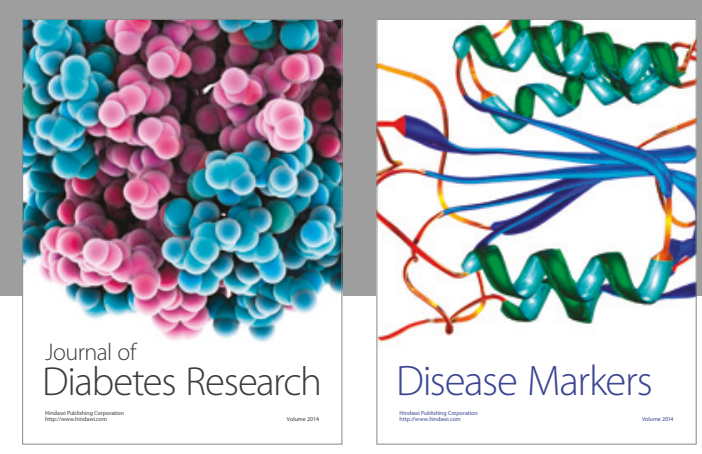

Disease Markers
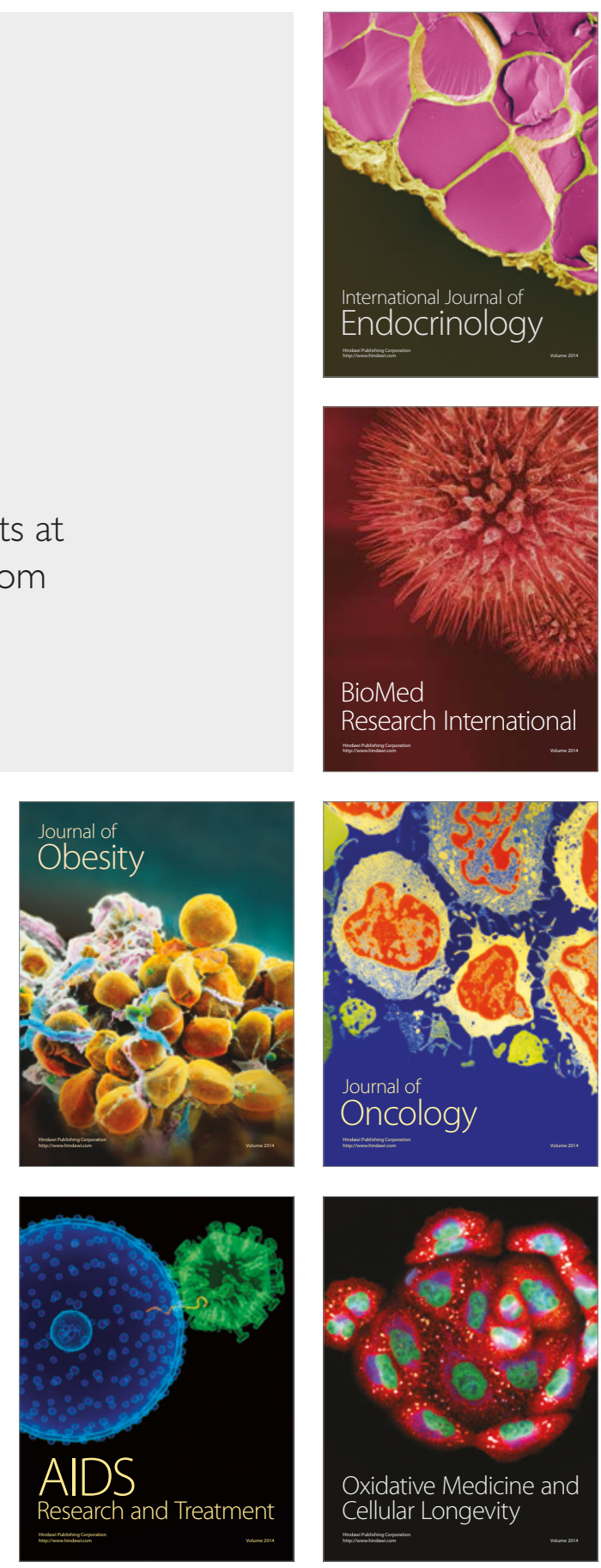